\title{
Resistance Training for Glycemic Control, Muscular Strength, and Lean Body Mass in Old Type 2 Diabetic Patients: A Meta-Analysis
}

\author{
JungHoon Lee $\cdot$ DoHoun Kim $\cdot$ ChangKeun Kim
}

Received: January 24, 2017 / Published online: April 5, 2017

(C) The Author(s) 2017. This article is an open access publication

\section{ABSTRACT}

Introduction: Type 2 diabetes (T2D) in elderly patients is associated with accelerated loss of skeletal muscle mass and strength. However, there are few meta-analysis reviews which investigate the effects of resistance training (RT) on glycemic control and skeletal muscle in the patients.

Methods: Three electronic databases were searched (from the earliest date available to November 2016). Studies were included according to the inclusion criteria: T2D patients at least 60 years old, fasting plasma glucose of at least 7.0, and at least 8 weeks of RT.

Results: Fifteen cohorts of eight studies (360 patients, average age 66 years) met the inclusion criteria. RT groups lowered glycosylated hemoglobin (HbA1c) (mean $\mathrm{ES}=-0.37, \quad 95 \%$ $\mathrm{CI}=-0.55$ to $-0.20, P<0.01)$ but did not result

Enhanced content To view enhanced content for this article go to http://www.medengine.com/Redeem/ 8708F06044E00CB3.

J. Lee $(\bowtie) \cdot$ C. Kim

Department of Exercise Physiology, Korea National Sport University, Oryung-dong, Songpa-gu, Seoul, Republic of Korea

e-mail: hoonlee518@gmail.com

D. Kim

Department of Nutrition, Food and Exercise Sciences, College of Human Sciences, Florida State University, Tallahassee, FL, USA in a significant effect on lean body mass (LBM) (mean $\mathrm{ES}=0.08, \quad 95 \% \mathrm{CI}=-0.15$ to 0.30 , $P=0.50)$. Homogeneity was shown between studies regarding $\mathrm{HbA} 1 \mathrm{c}$ and $\mathrm{LBM}(Q=15.70$, $d f=9, P=0.07$ and $Q=0.12, d f=4, P=0.998$, respectively). High-intensity subgroups showed a slight tendency to improve (rather than duration, frequency, and weekly volume) and to decrease HbA1c levels more than low-intensity subgroups $(P=0.37)$. RT increased muscular strength (mean $\mathrm{ES}=1.05,95 \% \mathrm{CI}=0.26-1.84$, $P=0.01$ ). No training components explained the heterogeneity between studies with changes in muscle strength.

Conclusion: RT improves glycemic control and muscle strength in elderly patients with T2D. RT with high intensity can be a strategy to treat patients with T2D and sarcopenia associated with aging.

Keywords: Aging; Glycosylated hemoglobin; Insulin sensitivity; Sarcopenia

\section{INTRODUCTION}

The prevalence of type 2 diabetes (T2D) has rapidly increased during the last two decades. In the USA, there were 7.8 million people diagnosed with diabetes in 1993. This number had risen to 29.1 million in 2012, which accounts for $9.3 \%$ of the total US population. Diabetes prevalence in the elderly is higher than in the 
younger population; $26 \%$ of those aged 65 years or older had diabetes, of which $90 \%$ to $95 \%$ have T2D [1].

T2D is characterized by hyperglycemia in the context of insulin resistance and impaired insulin secretion. The mechanism behind high susceptibility to T2D in the elderly still remains unclear. However, it has been suggested that alteration of lifestyle factors in the elderly, such as decreased physical activity and increased abdominal obesity, mainly contribute to developing insulin resistance [2]. In addition to insulin resistance in the body, elderly people with T2D are susceptible to diabetic-related physical frailty. Impaired physical function, decreased muscle strength and mass, and a high risk of falls and fractures are often reported for adults with diabetes [3-5]. Even though a reduction in skeletal muscle mass and increase in fat mass are common and consistent alterations in the body associated with ageing [6], T2D in older adults is associated with accelerated loss of skeletal muscle mass and strength [7]. As skeletal muscles are the major tissue for glucose uptake and clearance from the blood [8], reduction of muscle mass may aggravate insulin resistance in the elderly. In addition, increasing frailty along with decreased muscle strength may lead to a decrease in independence in elderly diabetics; the treatment regimens for older diabetic individuals need to improve insulin sensitivity and skeletal muscle mass and function.

Various forms of physical activity have been applied in the treatment of T2D. Beneficial effects of exercise training on glycemic control have been well established [9]. Exercise training improves glucose clearance rate [10] and reduces visceral adipose tissues, which seems to be related to insulin sensitivity [11]. Moreover, exercise training is beneficial to improve insulin sensitivity, independently of weight reduction [12].

Aerobic training (AT) has conventionally been recommended as the most suitable exercise type in the treatment for patients with T2D [13]. However, the significance of RT exercise has recently been brought up by many researchers $[10,14,15]$, and also the American College of Medicine (ACSM) has suggested incorporation of progressive RT in an exercise program for individuals with T2D [16]. Cauza et al. reported that 16 weeks of RT in middle-aged T2D patients improved HbA1c levels [17], and the same improvement of insulin sensitivity was reported in diabetic patients after 4-6 weeks moderate-intensity RT, and improved muscle strength was reported in the same group [18]. It is reported that supervised strength training in T2D patients for 4 months significantly increased quadriceps size as well as the muscle to fat ratio, indicating the improvement of muscle quality [19]. As suggested in the above studies, RT is shown to improve not only glycemic control but also skeletal muscle strength and mass. Benefits of resistance exercise training have been studied mostly in middle-aged T2D patients $[17,18]$, as do the systematic meta-analysis reviews $[20,21]$. Therefore, the main aim of this meta-analysis review was to evaluate and provide appropriate guidance for RT programs for older individuals with T2D. To achieve this, we systematically reviewed and quantified the effect of RT on HbA1c as well as muscular strength and lean body mass (LBM) in the elderly with T2D. We also investigated if the effects of RT are influenced by the training program variables such as intensity, duration, frequency, and weekly volume of exercise.

\section{METHODS}

\section{Study Selection}

This current meta-analysis review followed the strategy of The PRISMA Statement [22]. Searches of EMBASE, PubMed, and SportDiscus databases were performed for studies published in English from the earliest date available to November 2016. Manual searches of reference lists were conducted to ensure that all relevant studies were captured. Two reviewers (JHL and DHK) independently searched all of the articles and applied the inclusion and exclusion criteria to the titles and abstracts searched. Disagreements about the inclusion and exclusion were resolved by consultation or by another reviewer (CKK). When the information was not clear, the 
full-text papers of the studies were obtained for review. Corresponding authors of potentially eligible studies were contacted if studies reported data for which it was impossible to discriminate.

Searches included terms such as "glycosylated hemoglobin or HbA1c", "resistance training or strength training", and "type 2 diabetes". Participants were at least 60 years old and had T2D, which was defined by the World Health Organization and the American Diabetes Association's criterion of fasting plasma glucose of at least $7.0 \mathrm{mmol} / \mathrm{l}$. Included RT was required to have a duration of at least 8 weeks because our main outcome of interest, HbA1c, reflects average blood glucose concentration from the previous $8-12$ weeks. Trials including combined or mixed training and dietary supplements were excluded to focus on the effects of RT alone. We included trials of RT that were structured and supervised as described in terms of duration, frequency, intensity, and type to quantify the training intervention and compliance except for one trial [control (CON) group of [23]; home-based RT]. Studies were excluded if the subjects were not human or patients had type 1 diabetes or gestational diabetes. Duplicate studies or sub-studies of included trials were also excluded from the analysis.

Two reviewers (JHL and DHK) independently, in addition to another reviewer (CKK), assessed the quality of the included studies using the PRISMA recommendations [22]. The assessment of quality consisted of six items: appropriate generation of random allocation sequence, concealment of the allocation sequence, blinding of the assessment and collection outcomes, proportion of participants lost to follow-up, complete outcome data, and the intention-to-treat principle [22]. The assessment was considered as a criterion to decide whether the selected studies were included or not.

\section{Data Extraction}

Data were extracted from all selected studies by two independent reviewers to record the detailed information regarding subject characteristics, study methods, interventions, outcomes, and adverse events. We used means and standard deviation (SD); where standard errors were provided, they were converted to SD. Corresponding authors were contacted for detailed information where required. In terms of population characteristics, age, gender, BMI, number, and duration of T2D of participants were recorded to compare the similarity of participants between trials.

The primary outcome was HbA1c levels and two secondary outcomes were muscular strength (\% change in the one-repetition maximum (1-RM) or dynamometry) and muscle mass (LBM or skeletal muscle mass). We selected only whole-body lean mass or whole-body skeletal muscle mass for LBM. If muscle strength was reported as upper and lower body, we used the lower body because the lower body has a larger storage space for glucose disposal than the upper body, which is more appropriate for T2D patients. In addition, development of lower body muscular strength is associated with advanced daily physical activity of the elderly [24].

Regarding intervention, we recorded total duration, frequency (times per week), intensity (\% of 1-RM), weekly volume (number of repetitions or sets $\times$ number of exercises $\times$ frequency), session duration, target muscles of exercise, names of exercise machine or tool, and training place to compare the similarity of training methods between trials. The mean values were used for calculation if the studies reported a range of data (e.g., 12.5, repetition 10-15). Detailed interventions about CON groups and any additional supplements were also recorded.

\section{Data Analysis}

Heterogeneity between studies was assessed using the Cochran $Q$ statistic [25]. In each study, the effect size (ES) for the intervention was calculated as the difference between the means of the post-measurement and pre-measurement at the end of the intervention using Hedges' $g$. Separate meta-analyses of trials with HbA1c, muscle strength, and muscle mass were performed to generate the mean ES and 95\% CI. ESs were classified according to Cohen's 
definition (1988), where 0.2 is considered small, 0.5 moderate, and 0.8 large [26]. We used a fixed-effects model when homogeneity was verified or a random-effects model when heterogeneity was shown by the $Q$ statistic. If the $Q$ statistic was significant $(P<0.05)$, which indicates heterogeneity of effects, we performed subgroups analyses [27]. All calculations were conducted with SPSS software version 20 .

\section{Compliance with Ethics Guidelines}

This article is based on previously conducted studies and does not involve any new studies of human or animal subjects performed by any of the authors.

\section{RESULTS}

\section{Flow of Studies Through the Review}

The search resulted in 15,437 potential studies. From the titles and abstracts, 15,413 studies were excluded on the basis of the exclusion criteria, and then 24 full-text studies were reviewed. Of these, 16 articles were excluded; three articles were duplicate $[15,28,29]$, five had participants under 60 years old $[17,30-33]$, two did not provide precise data $[34,35]$, two additionally treated with dietary supplements [36, 37], two involved combined exercise training [38, 39], and two involved a short-term exercise training $[40,41]$. The CON group of [42] was included in RT groups because it was a supervised low-intensity training group. The CON group of [23] conducted both supervised RT and home-based RT for 14 months. After discussing whether the CON group of [23] is included or not in RT groups, we decided that the CON group be included because they performed supervised RT for the first 2 months and then home-based RT for 10 months two or three times per week the same as with the supervised group. In addition, the number of home-based exercise sessions that the CON group did was recorded and they were encouraged to exercise by telephone and educated monthly. Eventually, we decided on a total of ten trials by consensus.

\section{Characteristics of Included Studies}

Table 1 shows the characteristics of all of the studies included. Articles were published from 1997 [29] to August 2013 [42]. The sample size was 391. Of these, 360 participants completed their intervention (RT, 241; CON, 119; female $\%, 47 \%$ ) ranging from 9 [13] to 100 [42] participants. The average age of the participants was 66 years (RT, $66 \pm 4.8$; CON, $66 \pm 2.8$ ). Mean BMI was 31.2 (RT, $31.3 \pm 4.1$; CON, $31.0 \pm 2.9$ ). Mean diabetes duration was 8.4 years (RT, $7.6 \pm 4.6$; CON, $9.2 \pm 2.9$ ). Mean baseline HbA1c was $7.8 \%$ (RT, $7.7 \pm 3.7$; CON, $7.9 \pm 7.1)$.

All ten trials of eight articles measured HbA1c as an index of glycemic control $[13,15,23,28,29,42-44]$. Five trials measured muscular strength by maximum weight moved $(1-\mathrm{RM})[13,15,28,43]$ or the number of repetitions or number of repetitions multiplied by the load [29]. We did not extract data about the variation in muscular strength from [28] for a meta-analysis because the study presented the results only in graphs with percentage changes, although we did contact the corresponding authors. Castaneda et al. [15] presented results as the sum of 1-RM measures for all machines used for training, Dunstan et al. [28] and Brooks et al. [43] measured upper and lower body muscular strength, respectively, Ibañez et al. [13] measured bench press and half squat, and Honkola et al. [29] measured the number of repetitions with given load-quadriceps muscles. However, as we described above, we chose the lower body rather than upper body. In terms of muscle mass, three trials measured whole-body lean mass by dual X-ray absorptiometry $[15,28,43]$ and one trial measured skeletal muscle by bioelectrical impedance analysis [42].

All interventions, except for the home-based training, were supervised in research centers using free weights or weight machines by instructors, research assistants, or physiotherapists. In all interventions, the mean training period was 26 weeks (minimum-maximum, 12 min [44]-56 [23] weeks). Mean session duration was $52 \mathrm{~min}$ (minimum-maximum, 45 min [15, 42, 43]-60 [44] min). All training 


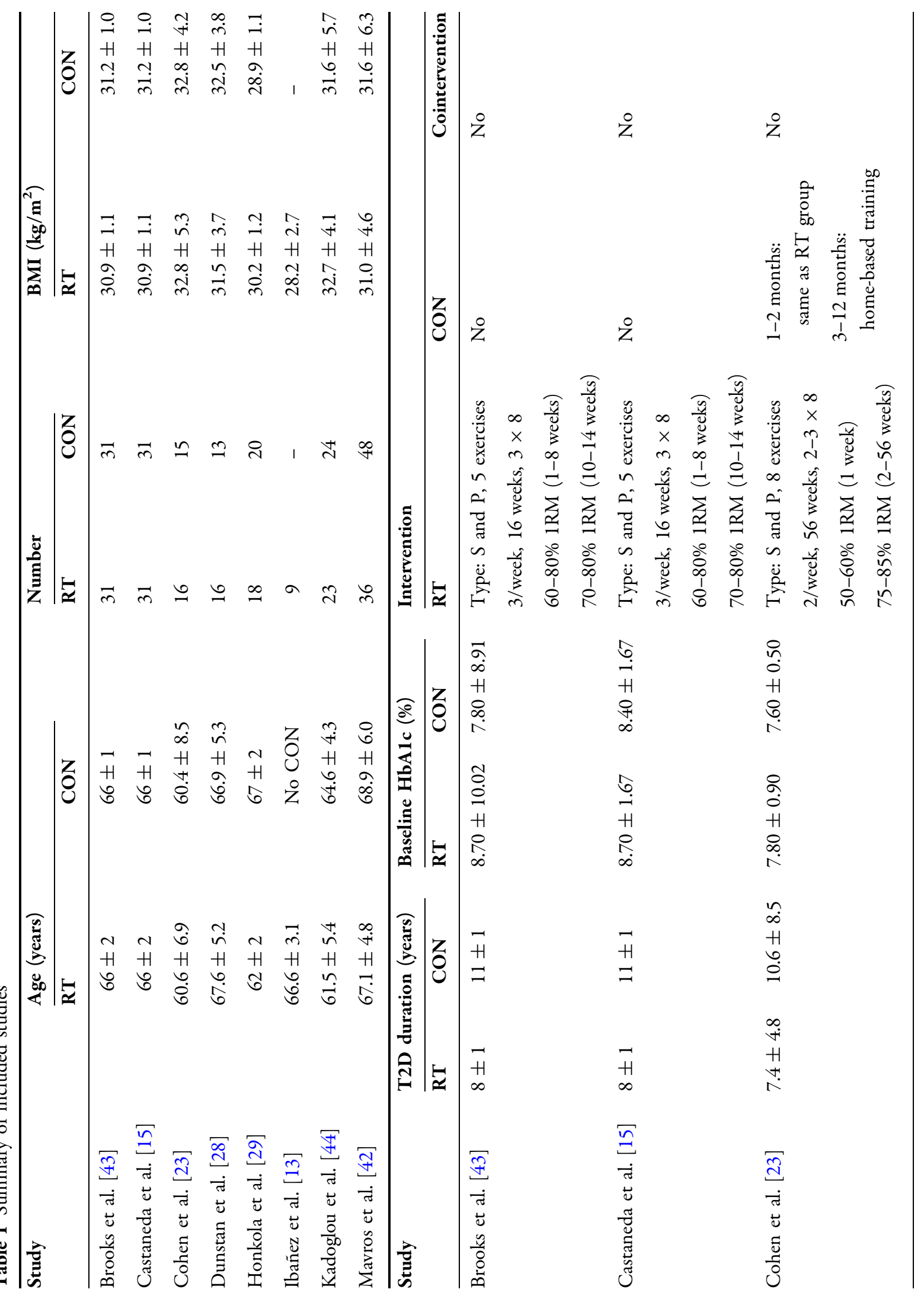




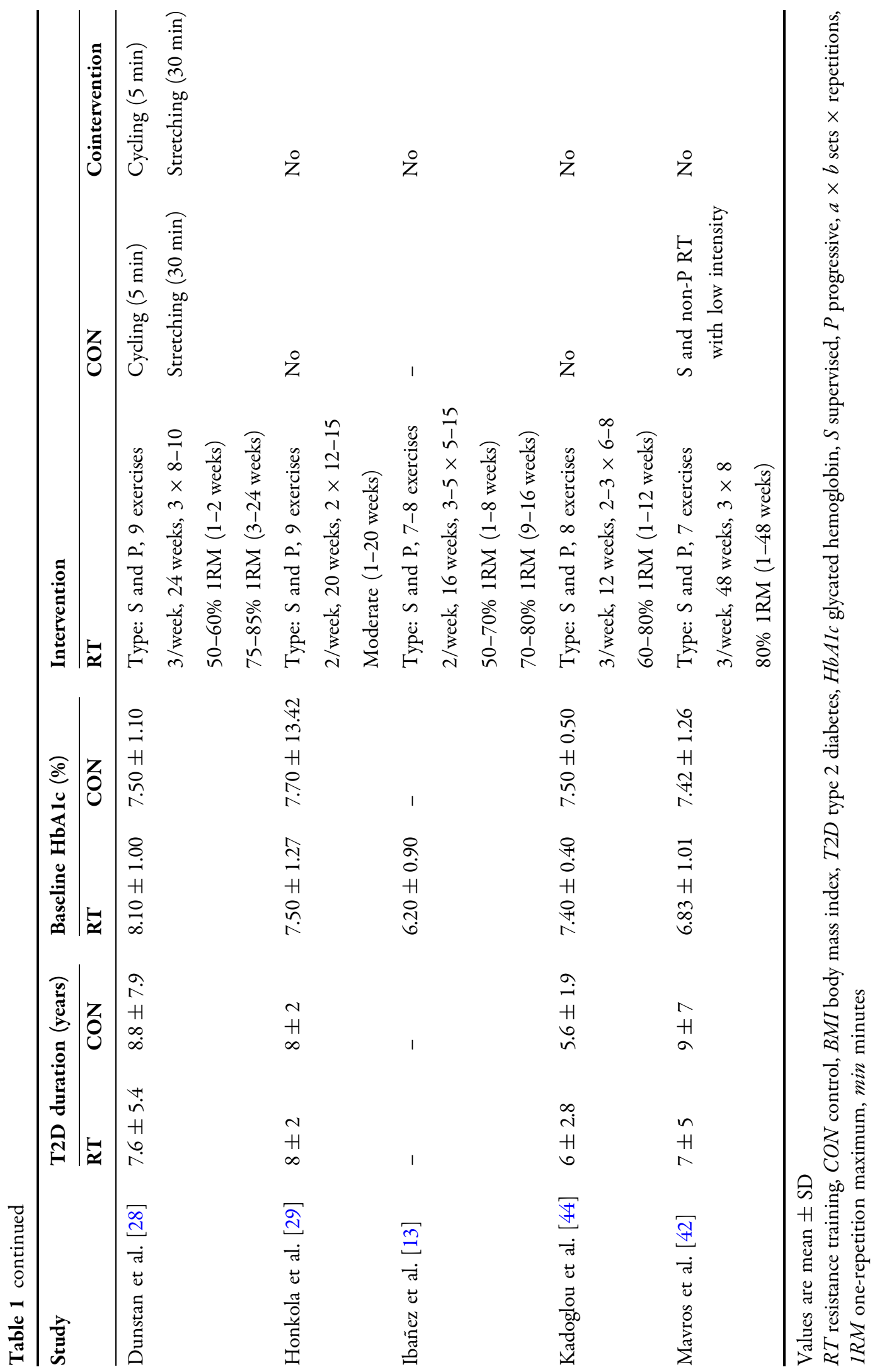


consisted of 2-3 sets (with the exception of [13] where $3-5$ sets were completed and home-based training of [23]), 8-15 repetitions, and 5-10 exercises. The number of total sets per week ranged from 36 [29] to 81 [28], and the number of total repetitions per week ranged from 320 [23] to 729 [28]. Intensities ranged from low (not specifically described in the CON groups of $[23,37])$ to moderate/high $(50-85 \%$ of the $1-\mathrm{RM}$ in the experimental groups of $[13,15,23,28,29,42-44])$. All training groups progressively increased their resistance up to $80-85 \%$ of $1-\mathrm{RM}$ over the duration of the intervention on the basis of the progression of 1-RM tested at baseline and mid-study except the CON groups of [23] and [42], but it is unsure whether the training groups were asked to exercise to the point of momentary muscular failure. Of the remaining two low-intensity groups, Mavros et al. [42] just reported that the CON group's resistance was set as low as possible and not progressed, but all the other things, such as exercise type, frequency, volume, and equipment, were the same as the experimental group. In [23], the home-based training group was supplied with one hand weight each and completed a standard upper body RT program in the home setting. We could not get further information about that, although we contacted two of the corresponding authors. In this current study, the CON groups consisted of non-exercise $[15,29,43,44]$ and aerobic training with static stretching exercises [28].

In the quality assessment, $88 \%$ reported appropriate generation of a random allocation sequence (7 of 8 ), 13\% presented concealment of the allocation sequence ( 1 of 8 ), 38\% described blinding of the assessment and collection outcomes (3 of 8), 100\% explained proportion of participants lost to follow-up (8 of 8 ), $50 \%$ exhibited complete outcome data (4 of 8 ), and $25 \%$ reported that the intention-to-treat principle was used for statistical analyses (2 of 8 ).

\section{Effect of Progressive Resistance Exercise}

\section{Glycosylated Hemoglobin}

RT in ten trials significantly lowered HbA1c (mean $\mathrm{ES}=-0.37,95 \% \mathrm{CI}=-0.55$ to -0.20 ,
$P<0.01$ ) (Fig. 1). The absolute reduction of HbA1c was $0.50 \%$. Univariate meta-regression did not show heterogeneity between studies $(Q=15.70, d f=9, P=0.07)$, and thus, we did not perform multivariate analysis with duration, intensity, frequency, and weekly volume variables. In other words, there were no correlations between RT's duration, intensity, session times per week, and weekly volume (the total number of sets and/or repetitions per week) and the changes in HbA1c levels.

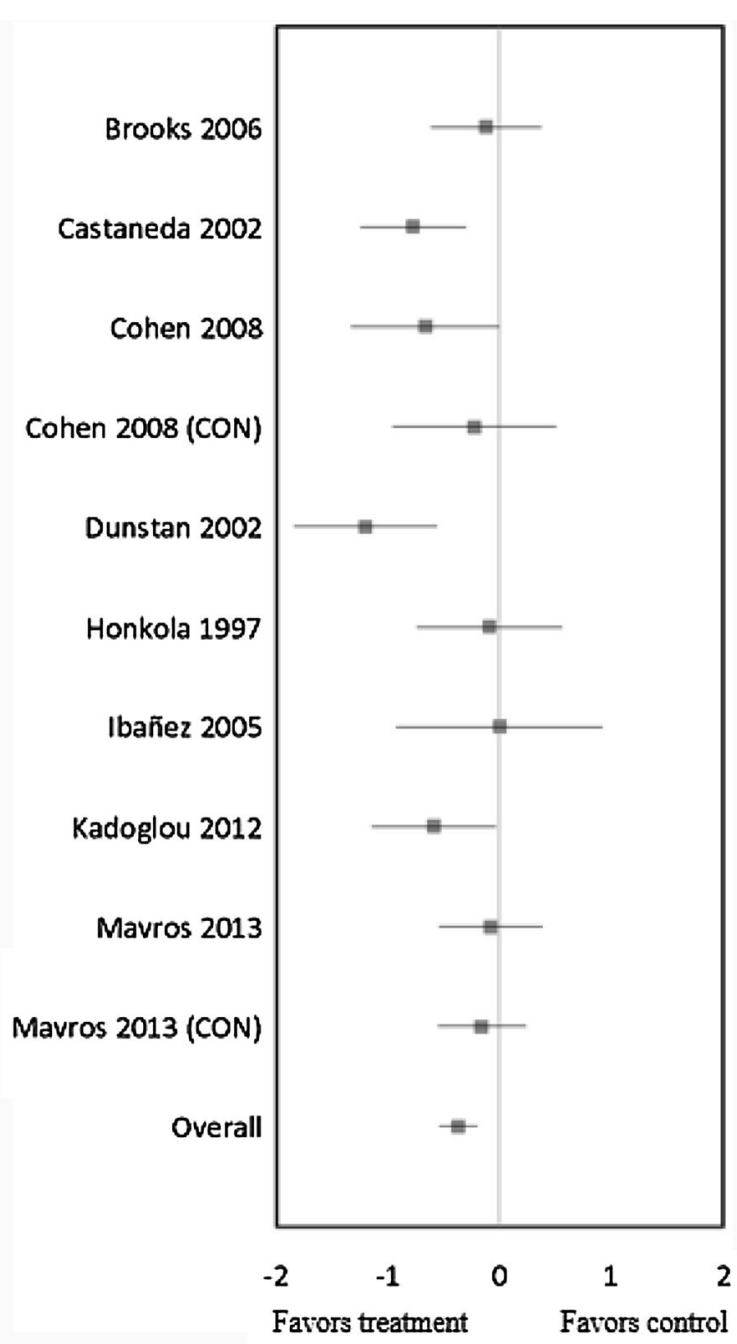

Fig. 1 Forest plot of effect sizes and 95\% confidence Intervals for all ten cohorts (8 studies) representing glycated hemoglobin, based on the fixed effects meta-analysis results. $C O N$ control group 


\section{Muscular Strength}

RT significantly increased muscular strength in four trials (mean $\mathrm{ES}=1.05,95 \% \mathrm{CI}=0.26$ to $1.84, P=0.01$ ) (Fig. 2). The relative increase of strength was $38 \%$, which included the results from [28] presenting only percentage changes. Univariate meta-regression showed heterogeneity between studies $(Q=17.79, d f=3$, $P<0.01$ ), and thus, we did further multivariate analysis with duration, intensity, frequency, and weekly volume variables. However, there were no correlations between RT session times per week $(Q=0.06, d f=1, P=0.81)$ and weekly total number of repetitions $(Q=0.06, d f=1$, $P=0.81$ ) and the changes in strength levels. We excluded RT's duration, intensity, and weekly total number of sets for the multivariate analysis because all four trials performed at high intensity $[13,15,29,43]$, only one trial was

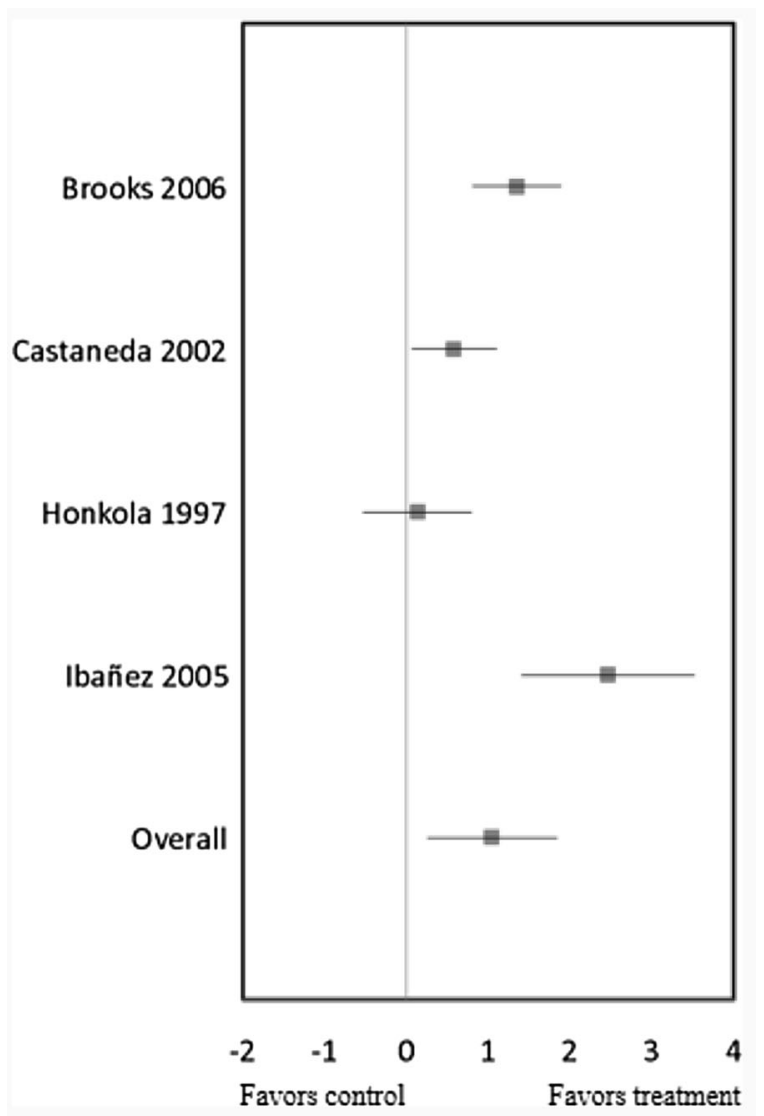

Fig. 2 Forest plot of effect sizes and 95\% confidence intervals for all 4 cohorts ( 4 studies) representing muscular strength, based on the random effects meta-analysis results conducted over the long term [29] and/or much higher volume per week [13], indicating that classifying into subgroups was impossible in the multivariate analysis.

\section{Lean Body Mass}

RT in five trials did not result in a significant effect on LBM (mean ES $=0.08,95 \% \mathrm{CI}=-0.15$ to $0.30, P=0.50$ ) (Fig. 3). The absolute increase of LBM was $0.78 \mathrm{~kg}$. Univariate meta-regression did not show heterogeneity between studies $(Q=0.12, d f=4, P=0.998)$, and thus, we did not perform multivariate analysis with duration, intensity, frequency, and weekly volume variables. In other words, there were no correlations between RT's duration, intensity, session times per week, and weekly volume (the total

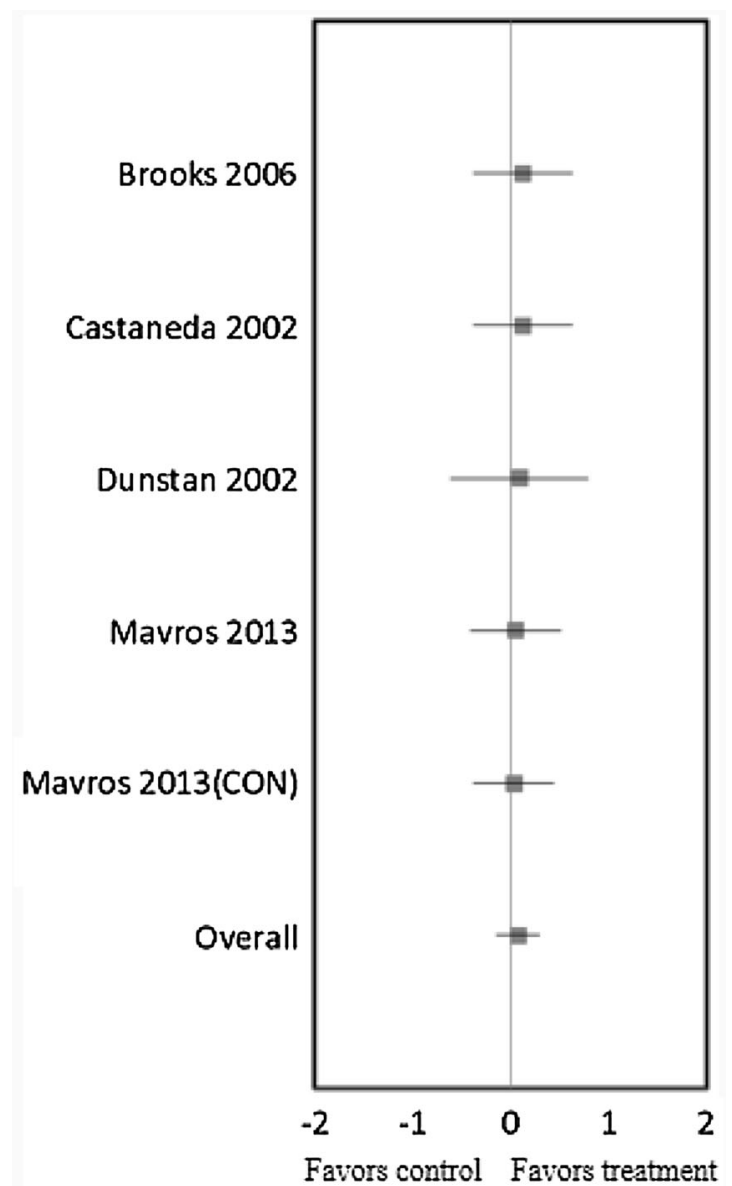

Fig. 3 Forest plot of effect sizes and 95\% confidence intervals for all five cohorts (4 studies) representing lean body mass, based on the fixed effects meta-analysis results. CON control group 
number of sets and/or repetitions per week) and the changes in LBM.

\section{Adverse Events}

The presence or absence of adverse events was recorded in four of the ten trials. Two of these reported that there were no adverse events $[29,44]$ and Ibañez et al. [13] stated that other than transient musculoskeletal soreness, no major complications or injuries were observed. The fourth trial, Castaneda et al. [15], reported that there were three incidents of chest pain in subjects with coronary artery disease and five mild hypoglycemic events, but no exercise-related injuries or adverse events in other subjects.

\section{DISCUSSION}

The primary results of this study are that RT significantly reduced $\mathrm{HbA} 1 \mathrm{c}$ by $0.50 \%$ and increased muscular strength by $38 \%$ in elderly patients with T2D, although there was no change in LBM.

In terms of HbA1c, we cannot explain the training effects by finding principal variables because there is homogeneity between studies. One possible interpretation is that different baseline HbA1c levels might affect the training effects as subgroups with high baseline HbA1c levels showed a slight tendency to decrease more than subgroups with low baseline HbA1c levels after training $(P=0.30)$. This result was supported by previous studies [20, 45-47]. In a previous review with a meta-analysis (mean age 58 years), no RT components were associated with changes in HbA1c, like this current review [20]. In contrast, Mavros et al. reported in their randomized study that only the high-intensity training group showed improvement of HbA1c levels, i.e., this improvement was not shown in the low-intensity RT group [42]. Therefore, it is unclear to date whether intensity, as well as other training components, is a paramount factor of RT for effectively improving HbA1c. However, the current review shows that the effect of RT on HbA1c levels is more closely influenced by exercise intensity in comparison with other training components such as duration, frequency, weekly volume 1 (total number of sets per week) and 2 (total number of repetitions per week) $(P=0.37, P=0.82, P=0.53$, $P=0.94$, and $P=0.58$, respectively). On the basis of these findings, we might be able to recommend that the elderly with T2D need to concentrate more on intensity than duration, frequency, and volume to improve both glycemic control and muscular strength all together. Gordon et al. [48] and Irvine et al. [49] suggested in their meta-analysis reviews that higher RT intensity may be a paramount factor to benefit glucose control, and the meta-analysis review by Peterson et al. also showed that high-intensity RT in healthy older adults increases muscle strength more than low-intensity RT (mean age 67 years) [50]. These results of RT meta-analysis reviews are contrary to those of an AT meta-analysis review which reported that the frequency of training may be more crucial for improving glycemic control than intensity [20].

There are several other important reasons why high-intensity RT might be necessary for the elderly with T2D. Two primary risk factors for development of T2D in the elderly are central obesity and physical inactivity [2]. Lack of physical activity is closely associated with the reduction of muscular strength and muscle mass, which may aggravate diabetes through decreased vascular supply and peripheral neuropathy [51]. In addition to that, decreased muscle mass compromises glycemic control as skeletal muscle plays an important role in glucose clearance from blood vessels and storage [52]. Moreover, muscular strength and muscle mass decrease with aging [53], so that it is important for diabetic patients, especially diabetic elders, to increase both their muscular strength and muscle mass through RT, in particular high-intensity training. High-intensity RT (70-90\% 1-RM) more effectively improved both muscular strength and muscle mass in the elderly as well as young people [54]. The greater increase of muscular strength through high-intensity RT possibly helps the elderly more effectively to improve their low physical activity, which is one of the risk factors for T2D. Central obesity in the elderly, another risk 
factor for T2D, may be alleviated more by high-intensity RT than by low-intensity RT [42]. This is probably attributed to high-intensity RT increasing fatty acid oxidation by promoting the oxidative function of mitochondria [55]. Furthermore, in a previous study, only high-intensity RT was shown to decrease HbA1c levels in the elderly whereas the low-intensity training did not in spite of both groups' increased muscle mass [42]. Castaneda et al. also reported that high-intensity training decreases HbA1c twice as much as moderate-intensity training because glucose uptake in skeletal muscle could be increased more at high intensity than at low or moderate intensity [15]. The majority of the trials in this current study conducted at high intensity reported no adverse effects other than transient musculoskeletal soreness and mild hypoglycemic events even though all subjects were untrained and had not previously participated in exercise training. Therefore, we should be able to recommend that elderly patients with T2D perform RT with high intensity rather than low or moderate intensity. However, high-intensity RT should be supervised by experienced instructors or exercise physiologists to ensure the safety of the elderly, and further studies are needed to determine whether high intensity is a primary training factor compared to other training components and whether the intensity is defined by high percentage of 1-RM or high degree of effort, such as training at low or moderate percentage of 1-RM until failure.

The decrease of muscle mass with aging is closely related to a decline in metabolic function [56] and glycemic control [13]. An increase in muscle mass can be equivalent to augmentation of the glucose reservoir [13] because the majority of insulin-stimulated glucose uptake occurs in skeletal muscle [57] and increased muscle mass can contribute to decreasing the secretion of insulin, which is necessary to maintain a normal glucose tolerance [52]. However, in our review, there is no change in LBM even though previous studies reported that increase in LBM is positively associated with the improvement of insulin sensitivity and glycemic control $[30,58]$ and that increased LBM may be a primary mediator of glucose homeostasis [28]. There are several possible reasons for our result. First, detailed information cannot be obtained regarding morphological changes, such as muscle volume, single fiber cross-sectional area, satellite cell concentration/differentiation, or architectural modifications through the measurement of LBM, so that it is not possible to determine the degree of hypertrophic/atrophic changes of muscle mass [59]. Second, given that carbohydrate, fat, and protein metabolism are interrupted in diabetes [60] and declined vascular supply and peripheral neuropathy may be the reasons why muscle mass loss is related to impaired glycemic control [51], T2D may have negative effects on the alterations of muscle mass. Lastly, no trials involved additional dietary supplements, such as protein, to promote muscle protein synthesis.

Such a result agrees with that of Irvine et al. in a previous meta-analysis review showing the reduction of HbA1c without increased LBM [49]. This is probably due to enhanced glucose clearance from the circulation through the increased number of glucose transporter-4 (GLUT4) and insulin receptors in skeletal muscle cells without an increase in muscle mass [44]. GLUT4 plays a crucial role in transporting glucose into tissues, and it is stimulated by muscle contraction and/or insulin in skeletal muscle tissues [61]. Other possible reasons for improved glycemic control without elevation of LBM are the improvement of intracellular insulin signaling [62] and the activation of glycogen synthase and protein kinase B by RT [43]. Moreover, RT is reported to reduce the concentration of plasma free fatty acid (FFA), which improves insulin sensitivity [43], as the decrease of plasma FFA levels leads to reduce intramyocellular triglyceride levels, which is correlated with improved insulin sensitivity [63].

Whether or not RT improves glycemic control more than AT is still controversial. In the current review, the absolute reduction of HbA1c is $0.50 \%$ in the RT groups. A meta-analysis review by Yang et al. reported that RT and AT decrease $\mathrm{HbA} 1 \mathrm{c}$ by $0.32 \%$ and $0.46 \%$, respectively [21]. A meta-analysis review by Umpierre et al. also showed that AT reduces HbA1c a little more than RT does (AT, 0.73\%; RT, 0.57\%) [47]. 
However, other previous studies reported that the effects of RT on glycemic control may be similar to those of AT $[49,64,65]$. The combined training effect (RT + AT) may also be controversial because Snowling and Hopkins did not find more benefits from combined training regarding glycemic control [66], whereas Sigal et al. reported that combined training significantly reduces HbA1c more than AT or RT alone [46]. We can probably explain the controversy in terms of the training methods variables, such as intensity, frequency, and duration, and the characteristics of participants, such as age, BMI, and levels of baseline HbA1c. These variables make it difficult to compare RT, $\mathrm{AT}$, and combined training in terms of effectiveness for glycemic control.

RT may improve glycemic control through a different physiological mechanism from AT [67]. RT plays an important role in increasing strength and mass of muscles, whereas AT is effective for reducing body fat by enhancing oxygen utilization [68]. Thus, elderly patients with T2D need to choose the type of training depending on their physical condition, purpose, and preference to improve their glycemic control. However, elderly patients with T2D may not have enough physical function to do AT with sufficient intensity and volume to improve glycemic control because they most likely are obese or have cardiovascular disease $[28,69]$. The American Diabetes Association recommended AT with moderate intensity for at least $150 \mathrm{~min}$ per week for diabetics [70]. Moreover, muscle weakness starts after the age of 50 [71] and the phenomenon worsens after the age of 65 [72], which aggravates impaired glycemic control in the elderly with T2D [51]. Therefore, for elderly patients, it would be helpful to know the importance of RT as a treatment method. However, on the basis of the finding in the current review, the extent of decreased absolute levels of HbA1c after RT is relatively small in the diabetic elderly. Thus, it could be recommended for diabetic patients to combine RT with other managements such as medical and/or dietary treatment for better results.

The strength of the study lies in its study design. First, this was the first meta-analysis study that investigated the effects of RT on glycemic control as well as strength and muscle mass in older T2D. Second, we included almost all previous RT studies that measured HbA1c as an index of glycemic control in elderly diabetic patients over the age of 60 . Lastly, we identified the importance of intensity of exercise training rather than duration, frequency, and volume for improving glycemic control and muscle strength and mass by separately analyzing the training variables. Thus, this study extends our knowledge to provide an effective therapeutic strategy to optimize both glycemic control and muscle strength and mass in the elderly with T2D.

However, there are some limitations in our study. First, the number of articles might not be adequate because we retrieved articles only published in English, which also has a potential publication bias. Only five of the ten trials measured muscle strength; among them one is not included because the study presented the results with only graphs, so that we did not get precise data; and four trials measured muscle mass by DEXA. Although we contacted several authors to obtain additional data or clarify issues about study design, we could not acquire the additional data regarding the secondary outcomes. Accordingly, there is a heterogeneity between studies regarding muscle strength; although homogeneity was presented between trials regarding $\mathrm{HbA1c}$ and muscle mass, no training variables explicitly explain the differences between subgroups. As all five trials conducted RT with similar intensity, we could not divide them into subgroups, such as low and high intensity regarding muscle strength. Second, we extracted the data of muscle strength from different parts, such as whole body [15], lower body [28, 43], and quadriceps muscles [29]; besides, four trials measured muscle strength by 1 -RM $[13,15,28,43]$ and one trial presented it as the number of repetitions multiplied by the load in kilograms [29]. Third, one study did not involve a CON group [13] and two studies had a low-intensity training group and home-based RT group as CON groups [23, 42]; we included these last two trials in the RT group, which may increase the risk of bias. However, several reviews reported that no 
differences exist in effect sizes between randomized and nonrandomized trials [73, 74].

\section{CONCLUSION}

This meta-analysis review suggests that RT significantly improved glycemic control and muscle strength in elderly patients with T2D, but there is no change in muscle mass. We might be able to recommend that the elderly with T2D need to place more emphasis on high intensity rather than duration, frequency, and volume to improve both glycemic control and muscular strength all together. Besides, RT can be safe for elderly diabetics on the basis of reports that there were rarely serious adverse effects in participants in the current review regardless of their previous training status. However, as there might be relatively small improvement of glycemic control with RT per se, in order for older individuals with type 2 diabetes to enhance the effects of RT on improvement of HbA1c levels, they should also include medical and/or dietary intervention into their treatment regimen along with RT. Future studies investigating training components in detail are warranted.

\section{ACKNOWLEDGEMENTS}

No funding or sponsorship was received for this study or publication of this article. All named authors meet the International Committee of Medical Journal Editors (ICMJE) criteria for authorship for this manuscript, take responsibility for the integrity of the work as a whole, and have given final approval for the version to be published. Analysis assistance in the preparation of this manuscript was provided by HyoJoon Yun of the Kinesmetrics and Sports Analysis Center of the Korea National Sport University.

Disclosures. JungHoon Lee, DoHoun Kim, and ChangKeun Kim declare that they have nothing to disclose.

Compliance with Ethics Guidelines. This article is based on previously conducted studies and does not involve any new studies of human or animal subjects performed by any of the authors.

Data Availability. The datasets during and/ or analyzed during the current study are available from the corresponding author on reasonable request.

Open Access. This article is distributed under the terms of the Creative Commons Attribution-NonCommercial 4.0 International License (http://creativecommons.org/licenses/ by-nc/4.0/), which permits any noncommercial use, distribution, and reproduction in any medium, provided you give appropriate credit to the original author(s) and the source, provide a link to the Creative Commons license, and indicate if changes were made.

\section{REFERENCES}

1. American Diabetes Association. National Diabetes Statistics Report. Estimates of diabetes and its burden in the epidemiologic estimation methods. Natl Diabetes Stat Rep. 2014;2014:2009-12.

2. Coon PJ, Rogus EM, Drinkwater D, Muller DC, Goldberg AP. Role of body fat distribution in the decline in insulin sensitivity and glucose tolerance with age. J Clin Endocrinol Metab. 1992;75(4):1125-32.

3. Gregg EW, Engelgau MM, Narayan V. Complications of diabetes in elderly people. BMJ. 2002;325:916-7.

4. Park SW, Goodpaster BH, Strotmeyer ES, et al. Decreased muscle strength and quality in older adults with type 2 diabetes: the health, aging, and body composition study. Diabetes. 2006;55(6): 1813-8.

5. Schwartz AV, Hillier TA, Sellmeyer DE, et al. Older women with diabetes have a higher risk of falls: a prospective study. Diabetes Care. 2002;25(10): 1749-54.

6. Fielding RA, Vellas B, Evans WJ, et al. Sarcopenia: an undiagnosed condition in older adults. Current consensus definition: prevalence, etiology, and consequences. International Working Group on Sarcopenia. J Am Med Dir Assoc. 2011;12(4): 249-56. 
7. Park SW, Goodpaster BH, Strotmeyer ES, et al. Accelerated loss of skeletal muscle strength in older adults with type 2 diabetes: the health, aging, and body composition study. Diabetes Care. 2007;30(6):1507-12.

8. DeFronzo R. Pathogenesis of type 2 diabetes mellitus. Med Clin N Am. 2004;88(4):787-835.

9. Borghouts LB, Keizer H. Exercise and insulin sensitivity: a review. Int J Sports Med. 2000;21(1):1-12.

10. Holten MK, Zacho M, Gaster M, Juel C, Wojtaszewski JFP, Dela F. Strength training increases insulin-mediated glucose uptake, GLUT4 content, and insulin signaling in skeletal muscle in patients with type 2 diabetes. Diabetes. 2004;53(2): 294-305.

11. Mourier A, Gautier JF, De Kerviler E, et al. Mobilization of visceral adipose tissue related to the improvement in insulin sensitivity in response to physical training in NIDDM. Effects of branched-chain amino acid supplements. Diabetes Care. 1997;20(3):385-91.

12. Venables MC, Jeukendrup AE. Aerobic training and obesity: effect on substrate metabolism and insulin sensitivity. Med Sci Sports Exerc. 2008;40(3): 495-502.

13. Ibañez J, Izquierdo $M$, Argüelles I, et al. Twiceweekly progressive resistance training decreases abdominal fat and improves insulin sensitivity in older men with type 2 diabetes. Diabetes Care. $2005 ; 28(3): 662-7$.

14. Dreyer HC, Fujita S, Cadenas JG, Chinkes DL, Volpi E, Rasmussen BB. Resistance exercise increases AMPK activity and reduces 4E-BP1 phosphorylation and protein synthesis in human skeletal muscle. J Physiol. 2006;576(Pt 2):613-24.

15. Castaneda C, Layne JE, Munoz-Orians L, et al. A randomized controlled trial of resistance exercise training to improve glycemic control in older adults with type 2 diabetes. Diabetes Care. 2002;25(12):2335-41.

16. Chodzko-Zajko WJ, Proctor DN, Fiatarone Singh M, et al. American College of Sports Medicine position stand. Exercise and physical activity for older adults. Med Sci Sports Exerc. 2009;41(7):1510-30.

17. Cauza E, Hanusch-Enserer U, Strasser B, et al. The relative benefits of endurance and strength training on the metabolic factors and muscle function of people with type 2 diabetes mellitus. Arch Phys Med Rehabil. 2005;86(8):1527-33.

18. Ishii T, Yamakita $\mathrm{T}$, Sato $\mathrm{T}$, Tanaka S, Fujii S. Resistance training improves insulin sensitivity in
NIDDM subjects without altering maximal oxygen uptake. Diabetes Care. 1998;21(8):1353-5.

19. Cauza E, Strehblow C, Metz-Schimmerl S, et al. Effects of progressive strength training on muscle mass in type 2 diabetes mellitus patients determined by computed tomography. Wien Med Wochenschr. 2009;159(5-6):141-7.

20. Umpierre D, Ribeiro PB, Schaan BD, Ribeiro JP. Volume of supervised exercise training impacts glycaemic control in patients with type 2 diabetes: a systematic review with meta-regression analysis. Diabetologia. 2013;56:242-51.

21. Yang Z, Scott CA, Mao C, Tang J, Farmer AJ. Resistance exercise versus aerobic exercise for type 2 diabetes: a systematic review and meta-analysis. Sports Med. 2014;44(4):487-99.

22. Liberati A, Altman DG, Tetzlaff J, et al. The PRISMA statement for reporting systematic reviews and meta-analyses of studies that evaluate health care interventions: explanation and elaboration. Ann Intern Med. 2009;151:W-65-94.

23. Cohen ND, Dunstan DW, Robinson C, Vulikh E, Zimmet PZ, Shaw JE. Improved endothelial function following a 14-month resistance exercise training program in adults with type 2 diabetes. Diabetes Res Clin Pract. 2008;79(3):405-11.

24. Srinivas-Shankar U, Roberts SA, Connolly MJ, et al. Effects of testosterone on muscle strength, physical function, body composition, and quality of life in intermediate-frail and frail elderly men: a randomized, double-blind, placebo-controlled study. J Clin Endocrinol Metab. 2010;95(2):639-50.

25. Cochran WG. Some methods for strengthening the common $\chi 2$ tests. Biometrics. 1954;10(4):417-51.

26. Cohen J. Statistical power analysis for the behavioral sciences (rev. ed.). Hillsdale: Lawrence Erlbaum Associates; 1988.

27. Higgins JPT, Thompson SG, Deeks JJ, Altman DG. Measuring inconsistency in meta-analyses. BMJ. 2003;327(7414):557-60.

28. Dunstan DW, Daly RM, Owen N, et al. High-intensity resistance training improves glycemic control in older patients with type 2 diabetes. Diabetes Care. 2002;25:1729-36.

29. Honkola A, Forsén T, Eriksson J. Resistance training improves the metabolic profile in individuals with type 2 diabetes. Acta Diabetol. 1997;34:245-8.

30. Baldi JC, Snowling N. Resistance training improves glycaemic control in obese type 2 diabetic men. Int J Sports Med. 2003;24(6):419-23. 
31. de Lade CG, Marins JC, Lima LM, et al. Effects of different exercise programs and minimal detectable changes in hemoglobin A1c in patients with type 2 diabetes. Diabetol Metab Syndr. 2016;8(13):1-9.

32. Fenicchia LM, Kanaley JA, Azevedo JL, et al. Influence of resistance exercise training on glucose control in women with type 2 diabetes. Metabolism. 2004;55(3):284-9.

33. Cauza E, Hanusch-Enserer U, Strasser B, et al. The metabolic effects of long term exercise in type 2 diabetes patients. Wien Med Wochenschr. 2006;156:515-9.

34. Dunstan DW, Daly RM, Owen N, et al. Home-based resistance training is not sufficient to maintain improved glycemic control following supervised training in older individuals with type 2 diabetes. Diabetes Care. 2005;28(1):3-9.

35. Simpson KA, Mavros Y, Kay S, et al. Graded resistance exercise and type 2 diabetes in older adults (the GREAT2DO study): methods and baseline cohort characteristics of a randomized controlled trial. Trials. 2015;16:512.

36. Geirsdottir OG, Arnarson A, Briem K, Ramel A, Jonsson PV, Thorsdottir I. Effect of 12-week resistance exercise program on body composition, muscle strength, physical function, and glucose metabolism in healthy, insulin-resistant, and diabetic elderly Icelanders. J Gerontol A Biol Sci Med Sci. 2012;67(11):1259-65.

37. Daly RM, Miller EG, Dunstan DW, et al. The effects of progressive resistance training combined with a whey-protein drink and vitamin D supplementation on glycaemic control, body composition and cardiometabolic risk factors in older adults with type 2 diabetes: study protocol for a randomized controlled trial. Trials. 2014;15:431.

38. Park SP, Lee IH. Effects on training and detraining on physical function, control of diabetes and anthropometrics in type 2 diabetes; a randomized controlled trial. Physiother Theory Pract. 2015;31(2):83-8.

39. Tan S, Li W, Wang J. Effects of six months of combined aerobic and resistance training for elderly patients with a long history of type 2 diabetes. J Sports Sci Med. 2012;11(3):495-501.

40. Gordon BA, Bird SR, MacIsaac RJ, Benson AC. Does a single bout of resistance or aerobic exercise after insulin dose reduction modulate glycaemic control in type 2 diabetes? A randomised cross-over trial. J Sci Med Sport. 2016;19(10):795-9.

41. Heden TD, Winn NC, Mari A, et al. Postdinner resistance exercise improves postprandial risk factors more effectively than predinner resistance exercise in patients with type 2 diabetes. J Appl Physiol. 2015;118(5):624-34.

42. Mavros Y, Kay S, Anderberg KA, et al. Changes in insulin resistance and $\mathrm{HbA} 1 \mathrm{c}$ are related to exercise-mediated changes in body composition in older adults with type 2 diabetes: interim outcomes from the GREAT2DO trial. Diabetes Care. 2013;36(8):2372-9.

43. Brooks N, Layne JE, Gordon PL, Roubenoff R, Nelson ME, Castaneda-Sceppa C. Strength training improves muscle quality and insulin sensitivity in Hispanic older adults with type 2 diabetes. Int J Med Sci. 2006;4(1):19-27.

44. Kadoglou NP, Fotiadis G, Athanasiadou Z, Vitta I, Lampropoulos $S$, Vrabas IS. The effects of resistance training on ApoB/ApoA-I ratio, Lp(a) and inflammatory markers in patients with type 2 diabetes. Endocrine. 2012;42(3):561-9.

45. Church TS, Blair SN, Cocreham S, et al. Effects of aerobic and resistance training on hemoglobin A1c levels in patients with type 2 diabetes: a randomized controlled trial. JAMA. 2010;304:2253-62.

46. Sigal RJ, Kenny GP, Boulé NG, et al. Effects of aerobic training, resistance training, or both on glycemic control in type 2 diabetes: a randomized trial. Ann Intern Med. 2007;147:357-69.

47. Umpierre D, Ribeiro PA, Kramer CK, et al. Physical activity advice only or structured exercise training and association with HbA1c levels in type 2 diabetes: a systematic review and meta-analysis. JAMA. 2011;305:1790-9.

48. Gordon BA, Benson AC, Bird SR, Fraser SF. Resistance training improves metabolic health in type 2 diabetes: a systematic review. Diabetes Res Clin Pract. 2009;83(2):157-75.

49. Irvine C, Taylor NF. Progressive resistance exercise improves glycaemic control in people with type 2 diabetes mellitus: a systematic review. Aust J Physiother. 2009;55(4):237-46.

50. Peterson MD, Rhea MR, Sen A, Gordon PM. Resistance exercise for muscular strength in older adults: a meta-analysis. Ageing Res Rev. 2010;9(3):226-37.

51. Schuller G, Linke A. Diabetes, exercise. In: Goldstein B, Muller-Wieland D, editors. Type 2 diabetes: principles, practice. New York: Informa Healthcare; 2008. Chap 6.

52. Miller WJ, Sherman WM, Ivy JL. Effect of strength training on glucose tolerance and post-glucose insulin response. Med Sci Sports Exerc. 1984;16:539-43. 
53. Evans WJ. Effects of exercise on body composition and functional capacity of the elderly. J Gerontol A Biol Sci Med Sci. 1995;50A:147-50.

54. Fielding RA. The role of progressive resistance training and nutrition in the preservation of lean body mass in the elderly. J Am Coll Nutr. 1995;14:587-94.

55. Tarnopolsky MA. Mitochondrial DNA shifting in older adults following resistance exercise training. Appl Physiol Nutr Metab. 2009;34:348-54.

56. Tzankoff SP, Norris AH. Effect of muscle mass decrease on agerelated BMR changes. J Appl Physiol. 1977;43:1001-6.

57. DeFronzo RA, Ferrannini E, Sato Y, Felig P, Wahren J. Synergistic interaction between exercise and insulin on peripheral glucose uptake. J Clin Investig. 1981;68:1468-74.

58. Cuff DJ, Meneilly GS, Martin A, Ignaszewski A, Tildesley HD, Frohlich JJ. Effective exercise modality to reduce insulin resistance in women with type 2 diabetes. Diabetes Care. 2003;26:2977-82.

59. Peterson MD, Sen A, Gordon PM. Influence of resistance exercise on lean body mass in aging adults: a meta-analysis. Med Sci Sports Exerc. 2011;43(2):249-58.

60. Zierath JR, Krook A, Wallberg-Henriksson H. Insulin action and insulin resistance in human skeletal muscle. Diabetologia. 2000;43:821-35.

61. Terada S, Yokozeki T, Kawanaka K, et al. Effects of high-intensity swimming training on GLUT-4 and glucose transport activity in rat skeletal muscle. J Appl Physiol. 2001;90(6):2019-24.

62. Tabata I, Suzuki Y, Fukunaga T, Yokozeki T, Akima $\mathrm{H}$, Funato K. Resistance training affects GLUT-4 content in skeletal muscle of humans after 19 days of head-down bed rest. J Appl Physiol. 1999;86:909-14.

63. Boden G, Shulman GI. Free fatty acids in obesity and type 2 diabetes: defining their role in the development of insulin resistance and beta-cell dysfunction. Eur J Clin Investig. 2002;32(Suppl 3):14-23.
64. Hurley B, Hagberg JM. Optimizing health in older persons: aerobic or strength training? Exerc Sports Sci Rev. 1998;26:61-89.

65. Taylor N, Dodd K, Shields N, Bruder A. Therapeutic exercise in physiotherapy practice is beneficial: a summary of systematic reviews 2002-2005. Aust J Physiother. 2007;53:7-16.

66. Snowling N, Hopkins W. Effects of different modes of exercise training on glucose control, risk factors for complications in type 2 diabetic patients: a meta-analysis. Diabetes Care. 2006;29:2518-27.

67. Poehlman ET, Dvorak RV, de Nino WF, Brochu M, Ades PA. Effects of resistance training and endurance training on insulin sensitivity in nonobese, young women: a randomized controlled trial. J Clin Endocrinol Metab. 2000;85:2463-8.

68. Plowman SA, Smith DL. Exercise physiology for health, fitness, and performance. 2nd ed. San Francisco: Benjamin Cummings; 2007.

69. Dela F, Kjaer M. Resistance training, insulin sensitivity and muscle function in the elderly. Essays Biochem. 2006;42:75-88.

70. Sigal RJ, Kenny GP, Wasserman DH, Castaneda-Sceppa C. Physical activity/exercise and type 2 diabetes. Diabetes Care. 2004;27(10):2518-39.

71. Lindle RS, Metter EJ, Lynch NA, et al. Age and gender comparisons of muscle strength in 654 women and men aged 20-93 yr. J Appl Physiol. 1997;83(5):1581-7.

72. Baumgartner RN, Koehler KM, Gallagher D, et al. Epidemiology of sarcopenia among the elderly in New Mexico. Am J Epidemiol. 1998;147(8):755-63.

73. Benson K, Hartz AJ. A comparison of observational studies and randomized, controlled trials. N Engl J Med. 2000;342:1878-86.

74. Concato J, Shah N, Horwitz RI. Randomized, controlled trials, observational studies, and the hierarchy of research designs. N Engl J Med. 2000;342: 1887-92. 